\title{
Congenital problems of mucociliary clearance: primary ciliary dyskinesia*
}

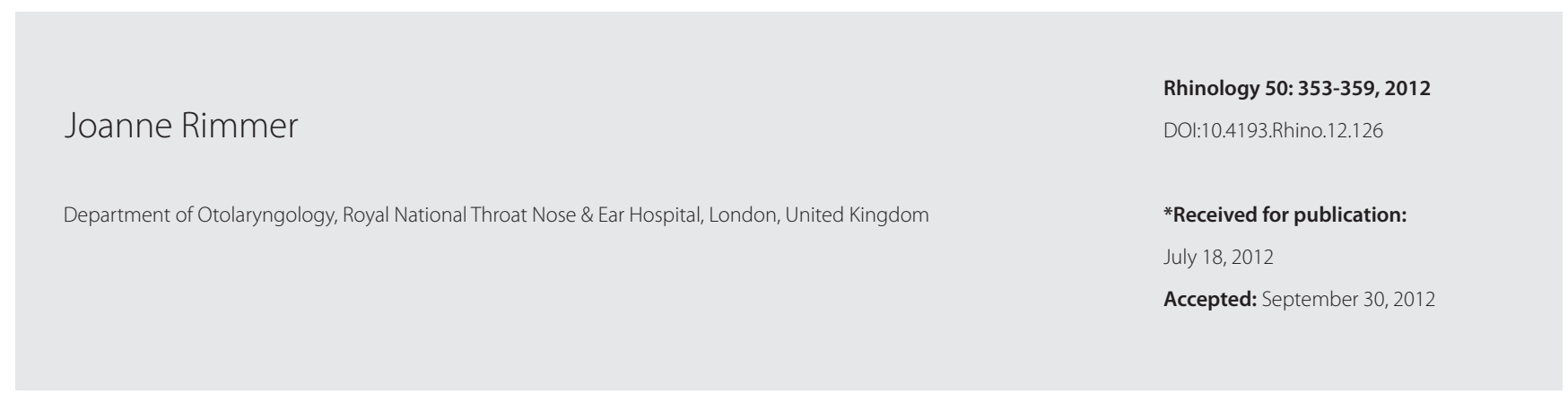

\section{Summary}

Mucociliary clearance is a primary defence mechanism of the airway that can be altered in congenital diseases such as primary ciliary dyskinesia and cystic fibrosis, as well as acquired conditions. This article focuses on primary ciliary dyskinesia and the diagnostic approach to it, which is still evolving.

Key words: mucociliary clearance, primary ciliary dyskinesia, cilia, Kartagener's syndrome

\section{Introduction}

Mucociliary clearance (MCC) is a primary defence mechanism of the airway, whereby secreted mucus and the substances within it are cleared ${ }^{(1)}$. It can be disturbed by infections, toxins and congenital disorders such as cystic fibrosis and primary ciliary dyskinesia (2).

Afzelius originally described ciliary ultrastructure in $1959^{(3)}$. He was the first to identify an ultrastructural abnormality in four men with 'living but immotile' sperm ${ }^{(4)}$. 'Immotile cilia syndrome' was formally described in 1977, in male patients with chronic respiratory symptoms, infertility and absent dynein arms ${ }^{(5)}$. As it has now been shown that cilia are not necessarily completely immotile, but may have abnormal motility, the name'primary ciliary dyskinesia' (PCD) seems a better alternative ${ }^{(6)}$. Afzelius also suggested that ciliary movement influences embryonic visceral rotation, and that in the absence of functional cilia this rotation will occur by chance ${ }^{(4)}$. Cilia are now known to be important in many organs during embryogenesis, and congenital ciliopathies may be associated with polycystic renal or hepatic disease, nephronophthisis, hydrocephalus and rare syndromes such as Bardet-Biedl, Alstrom's and oro-facial-digital syndromes ${ }^{(7)}$. Whilst motile cilia are a vital part of primary respiratory defence systems, it is sensory cilia that appear important in the development of these other organ systems.

\section{Mucociliary clearance}

Respiratory epithelium consists of approximately $80 \%$ ciliated cells and $20 \%$ goblet cells ${ }^{(8)}$. Airway surface fluid has two layers, an outer mucus 'gel' layer and an inner thin 'sol' layer ${ }^{(9)}$. This classical model has been challenged with an alternative model of a tangled mucin layer extending to the epithelial surface ${ }^{(10)}$. Mucus traps inhaled particles and pathogens and is then propelled by ciliary movement towards the gastrointestinal tract for elimination, the so-called 'mucociliary escalator.' The lower airways and nose can also clear mucus with a cough or a sneeze respectively, but the sinuses depend on mucociliary activity alone.

Effective MCC requires the production of appropriate mucus and periciliary fluid as well as coordinated ciliary activity ${ }^{(11)}$. Normal MCC has been reported as $4.5-7 \mathrm{~mm} /$ minute in the nose and 10.7 $\pm 3.5 \mathrm{~mm} /$ minute in the trachea and large lower airways ${ }^{(12,13)}$. 
Congenital problems of MCC may affect any part of this mechanism. PCD is a heterogeneous inherited disorder of ciliary function. Genetic mutations can affect mucus production, as in cystic fibrosis (CF) ${ }^{(9)}$. Acquired conditions such as chronic obstructive pulmonary disease may also reduce MCC, seemingly by depressing ciliary beat frequency, as can certain chemicals and toxins ${ }^{(14-16)}$.

\section{Ciliary structure and function}

Cilia are complex structures found on most eukaryotic cells. The lungs have $0.5 \mathrm{~m}^{2}$ of ciliated epithelium, containing $3 \times 10^{12}$ cilia; ciliated epithelium is also found in the nasal cavity and paranasal sinuses, Eustachian tubes and middle ear clefts, pharynx, trachea, Fallopian tubes and cervix, ductuli efferentes and the ependymal lining of the brain ${ }^{(9)}$.

Each ciliated epithelial cell carries approximately 200 cilia, each 5-7 $\mu \mathrm{m}$ in length. Axonemes form the core of cilia, and are composed of microtubules in specific arrangements by which cilia are classified. In motile cilia and spermatozoa tails, this is a ' $9+2$ ' arrangement: a ring of 9 outer microtubule pairs ('doublets') with a central pair of single microtubules ${ }^{(17)}$. The outer doublets are held to each other by nexin links, while radial spokes connect the central pair to each outer doublet (Figure 1). Immotile "primary" cilia have a ' $9+0$ ' arrangement i.e. they lack a central pair ${ }^{(18)}$. Embryonic nodal cilia, involved in left-right asymmetry, are also of this type.

The outer doublets carry inner and outer dynein arms ${ }^{(10)}$. Outer dynein arms (ODA) are multisubunit ATPase complexes that generate the energy required for ciliary movement ${ }^{(17)}$. The inner dynein arms (IDA) influence beat pattern ${ }^{(19)}$. Each cilium has a forward effective stroke followed by a backward recovery stroke (19-21). The central microtubule pair divides the axoneme into two halves; one side is active during the effective stroke and the other side during the recovery stroke ${ }^{(2)}$. Ciliary movement is coordinated by metachronal waves so that each ciliary beat is coupled to the adjacent cilium ${ }^{(22)}$. The direction of the effective stroke is determined by the orientation of the ciliary axoneme (11).

\section{Primary ciliary dyskinesia}

PCD is a rare genetic disorder, usually inherited in an autosomal recessive fashion. However, there are reported cases of autosomal dominant and $x$-linked inheritance ${ }^{(23-25)}$. It is a genetically heterogeneous ciliopathy characterized by abnormal structure and/or function of ' $9+2$ ' motile cilia and sperm flagella. Various ultrastructural defects have been described, and in some the genetic mutations have also been identified. Some patients have normal ciliary ultrastructure but abnormal random orientation or dyskinetic movement ${ }^{(26)}$.

The incidence has been quoted variably from 1:7.000 to 1:60.000 but is generally estimated now as approximately 1:15.000, higher in certain genetically isolated populations ${ }^{(17,18,27)}$. This is likely to be an underestimate due to difficulties in diagnosis $(7,18,28,29)$.

The clinical features are less heterogeneous than the genetic mutations. It may be apparent from birth, with unexplained neonatal respiratory distress seen in over $70 \%{ }^{(30)}$. Chronic respiratory infections are characteristic, with rhinitis, sinusitis and eventual bronchiectasis in nearly all patients. PCD should be considered in any 'atypical' asthma that does not respond to standard treatment ${ }^{(27)}$. Chronic rhinorrhoea is difficult to distinguish from common childhood upper respiratory infections, but any child with nasal polyps should be assessed for cystic fibrosis and then for PCD. There may be a persistent cough as patients attempt to clear secretions by a cilia-independent mechanism. Recurrent otitis media occurs in $95 \%$ of children, with chronic middle ear effusions causing hearing loss in a significant number ${ }^{(29)}$. Both men and women are subfertile rather than infertile. In men this is due to abnormalities of sperm motility (although $50 \%$ have normal fertility), while in women the Fallopian tube cilia are dysfunctional; this can lead to a higher incidence of ectopic pregnancy as well as difficulty conceiving ${ }^{(31)}$.

Defective function of embryonic nodal cilia leads to apparent randomisation of left-right axis development, with situs inversus found in almost $50 \%$ of PCD patients ${ }^{(31)}$. PCD with situs inversus is classically known as Kartagener's syndrome, although Siewert

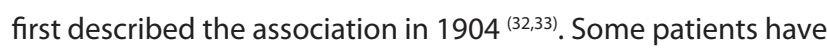
situs ambiguous or heterotaxy, where organs are in mirrored

Table 1. Common screening tests for PCD: tertiary referral for further investigation is advised if abnormal.

\begin{tabular}{|c|c|}
\hline Screening Test & Normal range \\
\hline Saccharin test & 10 - 20 mins; abnormal if $>60$ mins $(17,42,43)$ \\
\hline${ }^{99 m}$ Technetium-colloid scintigraphy & $4-11 \mathrm{~mm} / \mathrm{min}$; abnormal if $<3 \mathrm{~mm} / \mathrm{min}{ }^{(22,45)}$ \\
\hline Expired nasal NO & $\begin{array}{l}>500 p p b \text {; consider PCD if }<250 \mathrm{ppb} \text { and high clini- } \\
\text { cal suspicion; PCD highly likely if }<100 \mathrm{ppb}^{(50,51)}\end{array}$ \\
\hline
\end{tabular}


a

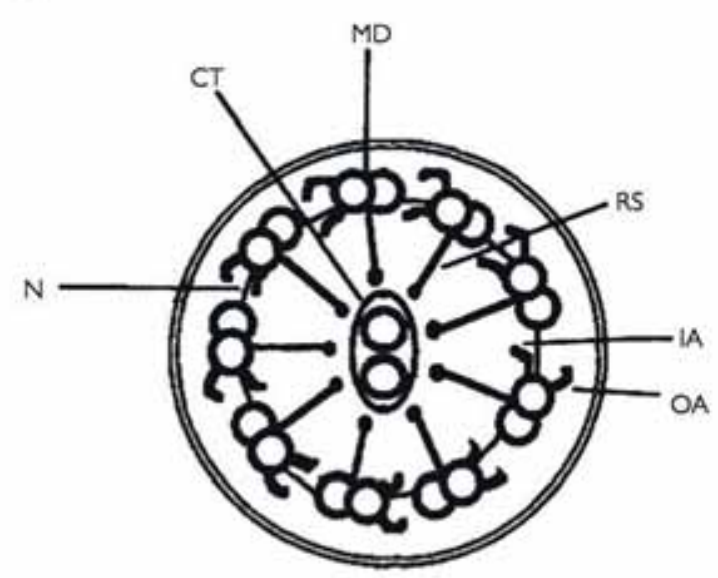

b

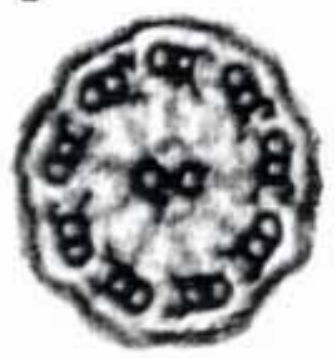

c

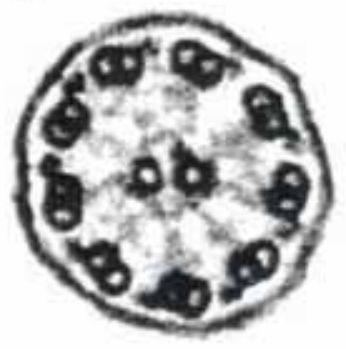

Figure 1. a) Schematic cross-section of a normal cilium: nine microtubule doublets (MD) are connected to two central microtubules (CT) by radial spokes (RS). Each doublet is connected to the next by a nexin link (N). Outer (OA) and inner (IA) dynein arms project from each doublet. b) Transmission electron micrograph of normal ciliary cross-section (magnification: $\times 33.000)$ c) Transmission electron micrograph of cross-section of cilium with absent inner dynein arms, in a patient with PCD (magnification: x33000)

Reprinted from Paediatric Respiratory Review, 1(1), Chilvers MA and O'Callaghan C, Local mucociliary defence mechanisms, p29, Copyright (2000), with permission from Elsevier. positions or even duplicated; this is often associated with congenital heart defects ${ }^{(34)}$.

The presentation of PCD is usually in mid-childhood and is often non-specific. Diagnosis is therefore frequently delayed, with resultant progression of respiratory disease. The European Respiratory Society's Task Force on PCD found that the median age at diagnosis was 5.3 years; this was lower in patients with situs inversus (3.5 years) compared to those without (5.8 years) ${ }^{(35)}$. Early diagnosis improves prognosis with stabilisation of lung function and subsequent reduction in longterm morbidity ${ }^{(36)}$. Any child with situs inversus should be investigated, as $25 \%$ will have PCD ${ }^{(37)}$. Similarly any child with PCD should have a cardiological evaluation, for they are more likely to have congenital heart disease (6\%) ${ }^{(34)}$. Other situs anomalies and any congenital heart defect should also prompt investigation for PCD ${ }^{(38)}$.

The genetics of ciliopathies are complex because of the multiple proteins involved in axonemal structure ${ }^{(20)}$. Eight PCD genes have been identified to date: DNAI1, DNAH5, DNAH11, DNAI2, TXNDC3, KTU, RSPH9 and RSPH4A ${ }^{(7,20)}$. DNAH5 is thought to account for approximately $28 \%$ of PCD cases, with DNAI1 causing a further $2-10 \%{ }^{(39,40)}$. The other genes have only been found in isolated or rare cases, so the genetic basis is unknown in over $60 \%$ of PCD cases $^{(7)}$. Several other loci have also been identified, including the retinitis pigmentosa gene and orofacial-digital type 1 syndrome gene, both found on the $X$ chromosome ${ }^{(18,25,31)}$.
As more PCD families are studied it is likely that other genes will be identified and potentially added to a screening panel ${ }^{(38)}$.

\section{Diagnosis}

First, more common conditions such as cystic fibrosis and congenital immune deficiencies should be excluded. Next, specific tests of ciliary structure and function should be performed; many of these are difficult, expensive and time-consuming, and should ideally be performed only in tertiary referral centres. The establishment of three such centres in the UK has significantly increased the percentage of positive diagnoses obtained, from $10 \%$ to $15 \%{ }^{(27)}$. Screening tests are often used to determine which patients should be referred for more complex investigations (Table 1).

\section{Screening tests}

\section{Assessment of mucociliary clearance}

The saccharin test is an easy and cheap but now somewhat outdated test of nasal MCC. A $5 \mathrm{mg}$ saccharin tablet is placed on the inferior turbinate, $1 \mathrm{~cm}$ from the mucocutaneous junction (41). The time taken for the subject to report a sweet taste is recorded. An alternative is to use a dye and record the time taken to visualise the dye at the back of the pharynx ${ }^{(42)}$. Normal values are $10-20$ minutes, and the test is abnormal if the time taken is more than 60 minutes ${ }^{(17,42,43)}$. It is difficult to perform reliably in 
young children who tend to sniff, giving a false negative result, and is therefore not helpful in early diagnosis. False positives are also common, and there is great inter- and intra-individual variability, but it may still be used as a screening test ${ }^{(7)}$. Scintigraphy is performed with a droplet of ${ }^{99 m}$ technetiumcolloid particles placed on the inferior turbinate. Radioactive movement is recorded with a gamma camera for 10 minutes ${ }^{(44)}$. Normal values are reported as $4-11 \mathrm{~mm} /$ minute with less than $3 \mathrm{~mm} /$ minute considered abnormal ${ }^{(22,45)}$. It does not require patient cooperation and is not affected by coughing, crying, sniffing or sneezing ${ }^{(44)}$. It has been shown to be $100 \%$ sensitive but with low specificity (55\%) and can be used as a reliable screening test, even in infants ${ }^{(44,45)}$.

An experimental method being used to assess MCC in mice involves placement of rhodamine dye a fixed distance 'upstream' of a microdialysis probe ${ }^{(46)}$. Regular samples are obtained through the probe and fluorometric analysis is performed; MCC can then be calculated.

Measurement of tracheobronchial MCC via pulmonary radioaerosol MCC (PRMC) is a potential test ${ }^{(47)}$. Nebulized ${ }^{99 m}$ technetium-albumin colloid is inhaled and lung radioactivity is measured. PMRC is calculated using whole-lung retention after 1 hour and 2 hours and comparing to the expected rates. It has a high specificity and is useful in excluding the diagnosis in difficult cases. However, the possibility of false positives with other pulmonary conditions remains. Cooperation is required and so it is unsuitable for children under 5 years.

MCC may also be studied in vitro. Adding $1 \mu \mathrm{m}$ diameter fluorescence beads to the apical surfaces of human airway epithelial air-liquid interface cultures allows direct visualisation with video epifluorescence or scanning confocal microscopy ${ }^{\left({ }^{(4)}\right)}$. Velocity and coordination of movement can be assessed.

\section{Expired nasal nitric oxide measurement}

Nitric oxide is synthesized in sinus epithelium by nitric oxide synthetase. Patients with PCD have very low expired nasal nitric oxide (nNO), although the reason for this remains unclear ${ }^{(49,50)}$.

This is very useful as a screening test; if $\mathrm{nNO}$ is within the normal range (greater than 250ppb) it excludes PCD with $97 \%$ certainty and has a $98 \%$ sensitivity ${ }^{(50,51)}$. Low levels do not confirm the diagnosis, as they are also seen in other congenital abnormalities of MCC as well as acquired conditions ${ }^{(7)}$. Air is ideally sampled during a breath-hold to avoid dilution from the lower airways, and this is difficult in children below six years of age ${ }^{(44)}$. However, alternative methods of sampling have been studied ${ }^{(52)}$. Expired nNO can be recorded during normal tidal breathing, when high levels would exclude PCD, but there is a lack of normal data for infants and children. Mean nNO in children under 1 year is significantly lower than in older cooperative subjects, presumably due to their undeveloped sinuses. Expired nNO may be used as a screening test even in young children but repeated measurements may be required ${ }^{(53)}$.

\section{Ciliary ultrastructural evaluation}

The current gold standard for diagnosis of PCD is ciliary ultrastructural evaluation using nasal brushings under transmission electron microscopy (TEM) ${ }^{(42,54)}$. However, up to $25 \%$ of PCD patients have normal ciliary ultrastructure so TEM is not infallible ${ }^{(27,54)}$. Combining ultrastructural evaluation with ciliary function assessment improves the accuracy of diagnosis but there remains a $10-25 \%$ misdiagnosis rate.

Various ultrastructural abnormalities have been reported in PCD: 24 - 43\% have isolated ODA defects; 7 - 45\% have defects of both inner and outer dynein arms; 11 - 30\% have IDA defects only; $7 \%$ have radial spoke abnormalities; $14 \%$ have a transposition defect affecting the central pair; and 3-6\% have complete ciliary aplasia ${ }^{(18,19,42,49)}$.

Immunofluorescent staining using antibodies to specific proteins enables dynein arm defects to be identified ${ }^{(55)}$. This newer test is relatively quick and inexpensive and may be of particular help in cases where the ultrastructural abnormality is difficult to detect with $\operatorname{TEM}^{(7,27,56)}$. Secondary ciliary abnormalities do not affect the result. As antibodies become available for other proteins this may become more useful.

\section{Ciliary function assessment}

Ciliary beat frequency (CBF) is the basic functional ciliary parameter that, with ciliary beat pattern (CBP), can be measured in vivo, ex vivo or in culture. Basal CBF is variably reported as

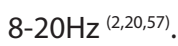

Previously, the best method available for analysis of CBF and CBP was high-speed cinematography. Advances in digital high speed video (DHSV) recording now allow up to 400 frames per second to be captured and analysed using special software. DHSV provides frame-by-frame views in three planes that allow CBP to be evaluated, although this technique is very time-consuming and there may be operator bias as only certain regions of beating cilia are selected for analysis ${ }^{(21)}$. Whole-field analysis of CBF avoids potential operator bias as there is no need to select certain fields for analysis, instead using software to assess the entire field at once ${ }^{(58)}$. This method is also much quicker than standard DHSV analysis, with no difference seen in CBF when the two techniques are compared ${ }^{(59)}$. Up to $25 \%$ of cases have dysfunctional cilia with apparently normal ultrastructure on TEM ${ }^{(42)}$. Some may have an abnormality in ciliary orientation so that the CBP is uncoordinated ${ }^{(27,60)}$. Different ultrastructural defects have been shown to produce predictable abnormal beat patterns ${ }^{(19)}$. Most cilia are virtually immotile in outer or combined inner/outer dynein arm defects. In inner arm defects there is an abnormal CBP with reduced amplitude. Central pair abnormalities cause a circular ciliary movement ${ }^{(61)}$. 
CBF and CBP are ideally measured using living cells immediately after removal from the nose ${ }^{(45,52)}$. If there has been a recent respiratory infection, secondary ciliary abnormalities may confuse the results and the tests may need repeating after a few weeks $(7,27,42)$. If assessment proves very difficult then in vitro ciliogenesis may be required. Jorissen et al. developed a sequential monolayer suspension culture technique that enables ciliogenesis ${ }^{(62)}$. This restores epithelial cells to their original ciliated phenotype, removing secondary defects; ultrastructure, $\mathrm{CBF}$ and $\mathrm{CBP}$ can then be reliably assessed ${ }^{(63)}$. It is $100 \%$ sensitive and specific, but culture is difficult to establish and therefore limited to a few centres worldwide ${ }^{(7,27)}$. It requires nasal biopsy, as brushings do not provide enough material, and takes six weeks, but it is a very accurate diagnostic test for PCD ${ }^{(54)}$.

\section{Genetic testing}

It is currently possible to test for the two most common mutations identified, DNAH5 and DNAI1, which account for 30-38\% of all PCD cases ${ }^{(20)}$. Because of the heterogeneity of PCD, this is really only useful in those with a known relative with PCD, either for diagnosis or carrier testing. The tests are currently only available in Freiburg, Germany and Chapel Hill, USA.

\section{Management}

Multidisciplinary care is essential for patients with PCD, with respiratory physicians and otolaryngologists involved, as well as physiotherapists.

Respiratory function is optimized with clearance of secretions using physiotherapy, postural drainage and exercise. Cough suppressants should be avoided ${ }^{(57)}$. Infective exacerbations should receive prompt antibiotic treatment, and prophylaxis with macrolides may be beneficial in established bronchiectasis. Non-invasive ventilation appears to improve quality of life and prolong survival, and successful lung transplantation has been reported ${ }^{(64)}$. Routine pneumococcal vaccination and annual influenza vaccinations are advised ${ }^{(7)}$.

'Masterly clinical inactivity' produces the best clinical outcomes as far as upper respiratory tract symptoms are concerned (7). Ventilation tubes should be avoided because of the almost inevitable persistent otorrhoea that follows. Hearing loss due to glue ear should instead be treated with hearing aids wherever possible, and tends to improve with age. Sinus surgery should be reserved for highly selected cases, including those with aggressive polyps; these are less common than in CF patients. Aggressive medical therapy, mainly in the form of saline douching, should be used to clear secretions from the nose and sinuses. Patients should be aware that they may be subfertile rather than infertile, and contraception should still be used. If conception is difficult, then fertility services should be available.

Genetic counseling should be offered to all PCD patients and their families.

\section{Conclusion}

PCD should be considered in patients with chronic intractable sinopulmonary symptoms, after excluding more common causes such as CF, immune deficiencies and Wegener's vasculitis. Patients with suspected PCD, or who have failed simple screening tests (Table 1), should be referred to a tertiary centre for evaluation and specialist diagnostic testing as appropriate. Management is currently symptomatic, and aims to prevent or delay the onset of bronchiectasis, but perhaps one day pharmacogene therapy may allow us to correct the ciliary dysfunction and restore mucociliary clearance.

\section{Conflict of Interest}

None to disclose.

\section{References}

1. Mall MA. Role of cilia, mucus, and airway surface liquid in mucociliary dysfunction: lessons from mouse models. J Aerosol Med Pulm Drug Deliv. 2008; 21: 13-24.

2. Stannard W, O'Callaghan C. Ciliary function and the role of cilia in clearance. J Aerosol Med. 2006; 19: 110-115.

3. Afzelius B. Electron microscopy of the sperm tail: results obtained with a new fixative. J Biophysic Biochem. 1959; 5: 269-278.

4. Afzelius BA. A human syndrome caused by immotile cilia. Science. 1976; 193: 317-319.

5. Eliasson R, Mossberg B, Camner P, Afzelius B. The immotile-cilia syndrome: a congenita ciliary abnormality as an etiological factor in chronic airway infection and male sterility. N Engl J Med. 1977; 297: 1-6.

6. Camner P. Minireview: how important is mucociliary clearance? Exp Lung Res. 1988; 14: 423-429.

7. Bush A, Hogg C, Mitchison H, Nisbet M, Wilson R. Update in primary ciliary dyskinesia. Clin Pulm Med. 2009; 16: 219-225.

8. Antunes MB, Cohen NA. Mucociliary clearance--a critical upper airway host defense mechanism and methods of assessment. Curr Opin Allergy Clin Immunol. 2007; 7: 5-10.

9. Houtmeyers E, Gosselink R, Gayan-Ramirez G, Decramer M. Regulation of mucociliary clearance in health and disease. Eur Respir J. 1999; 13: 1177-1188.

10. Boucher RC. Human airway ion transport. Am J Respir Crit Care Med. 1994; 150: 271 281.

11. Sleigh MA, Blake JR, Liron N. The propulsion of mucus by cilia. Am Rev Respir Dis. 1988;
137: 726-741.

12. Van der Baan S, Veerman AJP, Wulffraat $N$, et al. Primary ciliary dyskinesia: ciliary activity. Acta Otolaryngol. 1986; 102: 274-281.

13. Morgan L, Pearson $M$, de longh $R$, et al. Scintigraphic measurement of tracheal mucus velocity in vivo. Eur Resp J. 2004; 23: 518-522.

14. Koblizek V, Tomsova M, Cermakova E, et al. Impairment of nasal mucociliary clearance in former smokers with stable chronic obstructive pulmonary disease relates to the presence of a chronic bronchitis phenotype. Rhinology. 2011; 49: 397-406.

15. Yaghi A, Zaman A, Cox G, Dolovich MB. Ciliary beating is depressed in nasal cilia from chronic obstructive pulmonary disease subjects. Respir Med. 2012; 106: 11391142. 
16. Sommer JU, Stuck BA, Heiser C, Kassner SS, Hörmann K, Sadick H. Ciliary function of the nose in patients with Osler's disease and the effect of topically applied estrogens as a nose ointment. Rhinology. 2011; 49: 407-412.

17. Cowan MJ, Gladwin MT, Shelhamer JH. Disorders of ciliary motility. Am J Med Sci. 2001; 321: 3-10.

18. Escudier E, Duquesnoy P, Papon JF, Amselem S. Ciliary defects and genetics of primary ciliary dyskinesia. Paediatr Respir Rev. 2009; 10: 51-54.

19. Chilvers MA, Rutman A, O'Callaghan C. Ciliary beat pattern is associated with specific ultrastructural defects in primary ciliary dyskinesia. J Allergy Clin Immunol. 2003 112: 518-524

20. Leigh MW, Pittman JE, Carson JL, et al. Clinical and genetic aspects of primary ciliary dyskinesia/Kartagener syndrome. Genet Med 2009; 11: 473-87.

21. Chilvers M, O'Callaghan C. Analysis of ciliary beat pattern and beat frequency using digital high speed imaging: comparison with the photo-multiplier and photodiode methods. Thorax. 2000; 55: 314-317.

22. Jorissen M. Correlations among mucociliary transport, ciliary function, and ciliary structure. Am J Rhinol. 1998; 12: 53-58.

23. Narayan D, Krishnan SN, Upender M, et al. Unusual inheritance of primary ciliary dyskinesia (Kartagener's syndrome). J Med Genet. 1994; 31: 493-496

24. Krawczynski MR, Witt M. PCD and RP $X$-linked inheritance of both disorders? Pediatr Pulmon. 2004; 38: 88-89.

25. Moore A, Escudier E, Roger G, et al. RPGR is mutated in patients with a complex $X$ linked phenotype combining primary ciliary dyskinesia and retinitis pigmentosa. J Med Genet. 2006; 43: 326-333.

26. Buchdahl RM, Reiser J, Ingram D, Rutman A, Cole PJ, Warner JO. Ciliary abnormalities in respiratory disease. Arch Dis Child. 1988; 63: 238-243.

27. Hogg C. Primary ciliary dyskinesia: when to suspect the diagnosis and how to confirm it. Paediatr Respir Rev. 2009; 10: 44-50.

28. Chilvers MA, O'Callaghan C. Local mucociliary defence mechanisms. Paediatr Respir Rev. 2000; 1: 27-34.

29. Kennedy MP, Ostrowski LE. Primary ciliary dyskinesia and upper airway diseases. Curr Allergy Asthma Rep. 2006; 6: 513-517.

30. Holzmann D, Felix H. Neonatal respiratory distress syndrome - a sign of primary ciliary dyskinesia? Eur J Pediatr. 2000; 159: 857-860.

31. Castleman VH, Romio L, Chodhari R, et al. Mutations in radial spoke head protein genes RSPH9 and RSPH4A cause primary ciliary dyskinesia with central-microtubularpair abnormalities. Am J Hum Genet. 2009; 84: 197-209.

32. Kartagener M. Zur pathogenese der bronchiektasien. Beitr Klin Tuberk. 1933; 83: 489501.
33. Siewert AK. Über einen fall von bronchiec tasie bei einem patienten mit situs inversus viscerum. Berliner Klinische Wochenschrift. 1904; 41: 139-141.

34. Kennedy MP, Omran H, Leigh MW, et al Congenital heart disease and other het erotaxic defects in a large cohort of patients with primary ciliary dyskinesia. Circulation. 2007; 115: 2814-2821.

35. Kuehni CE, Frischer T, Strippoli MP, et al. ERS Task Force on Primary Ciliary Dyskinesia in Children. Factors influencing age at diagnosis of primary ciliary dyskinesia in European children. Eur Respir J. 2010; 36: 1248-1258.

36. Ellerman A, Bisgaard $H$. Longitudinal study of lung function in a cohort of primary ciliary dyskinesia. Eur Respir J. 1997; 10: 23762379

37. Kroon AA, Heij JMH, Kuijper WA, et al. Function and morphology of respiratory cilia in situs inversus. Clin Otolaryngol Allied Sci. 1991; 16: 294-297.

38. Morillas HN, Zariwala M, Knowles MR Genetic causes of bronchiectasis: primary ciliary dyskinesia. Respiration. 2007; 74: 252263.

39. Hornef N, Olbrich H, Horvath J, et al. DNAH5 mutations are a common cause of primary ciliary dyskinesia with outer dynein arm defects. Am J Respir Crit Care Med. 2006; 174: 120-126.

40. Zariwala MA, Leigh MW, Ceppa, F et al Mutations of DNAI1 in primary ciliary dyskinesia: evidence of founder effect in a common mutation. Am J Respir Crit Care Med. 2006; 174: 858-866.

41. Anderson I, Camner $\mathrm{P}$, Jensen $\mathrm{PL}$, Philipson $\mathrm{K}$, Proctor DF. Nasal clearance in monozygotic twins. Am Rev Respir Dis. 1974; 110: 301-305.

42. Jorissen M, Willems $T$, De Boeck K. Diagnostic evaluation of mucociliarytransport: from symptoms to coordinated ciliary activity after ciliogenesis in culture. Am Rhinol. 2000; 14: 345-352.

43. Stanley P, MacWilliam L, Greenstone M, et al. Efficacy of a saccharin test for screening to detect abnormal mucociliary clearance. $\mathrm{Br} J$ Dis Chest. 1984; 78: 62-65.

44. De Boeck K, Proesmans M, Mortelmans $L$, Van Billoen B, Willems $T$, Jorissen $M$. Mucociliary transport using $99 \mathrm{mTc}$-albumin colloid: a reliable screening test for primary ciliary dyskinesia. Thorax. 2005; 60: 414-417.

45. Armengot M, Milara J, Mata M, Carda C, Cortijo J. Cilia motility and structure in primary and secondary ciliary dyskinesia. Am J Rhinol Allergy. 2010; 24: 175-180.

46. Grubb BR, Jones JH, Boucher RC. Mucociliary transport determined by in vivo microdialysis in the airways of normal and CF mice. Am J Physiol Lung Cell Mol Physiol. 2004; 286: L588-L595.

47. Marthin JK, Mortensen J, Pressler T et al. Pulmonary radioaerosol mucociliary clearance in diagnosis of primary ciliary dyskinesia. Chest 2007; 132: 966-976.
48. Matsui H, Randell SH, Peretti SW, Davis CW, Boucher RC. Coordinated clearance of periciliary liquid and mucus from airway surfaces. J Clin Invest. 1998; 102: 1125-1131.

49. Noone PG, Leigh MW, Sannuti A, et al. Primary ciliary dyskinesia: diagnostic and phenotypic features. Am J Respir Crit Care Med. 2004; 169: 459-467.

50. Narang I, Ersu R, Wilson NM, Bush A. Nitric oxide in chronic airway inflammation in children: diagnostic use and pathophysiological significance. Thorax. 2002; 57: 586589

51. Wodehouse T, Kharitonov SA, Mackay IS, et al. Nasal nitric oxide measurements for the screening of primary ciliary dyskinesia. Eur Respir J. 2003; 21: 43-47.

52. Marthin JK. Nasal nitric oxide and pulmonary radioaerosol mucociliary clearance as supplementary tools in diagnosis of primary ciliary dyskinesia. Dan Med Bull. 2010; 57: 1-15.

53. Piacentini GL, Bodini A, Peroni D, et al. Nasal nitric oxide for early diagnosis of primary ciliary dyskinesia: practical issues in children. Respir Med. 2008; 102: 541-547.

54. Jorissen $M$, Willems $T$, Van der Schueren $B$. Ciliary function analysis for the diagnosis of primary ciliary dyskinesia: advantages of ciliogenesis in culture. Acta Otolaryngol. 2000; 120: 291-295.

55. Fliegauf M, Olbrich H, Horvath J, et al. Mislocalization of DNAH5 and DNAH9 in respiratory cells from patients with primary ciliary dyskinesia. Am J Respir Crit Care Med. 2005; 171: 1343-1349.

56. Zariwala MA, Knowles MR, Omran H. Genetic defects in ciliary structure and function. Annu Rev Physiol. 2007; 69: 423450.

57. Leigh MW. Primary ciliary dyskinesia. Semin Respir Crit Care Med. 2003; 24; 653-662.

58. Sisson JH, Stoner JA, Ammons BA, Wyatt TA. All-digital image capture and whole-field analysis of ciliary beat frequency. J Microsc. 2003: 211: 103-111.

59. Dimova S, Maes F, Brewster ME, et al. Highspeed digital imaging method for ciliary beat frequency measurement. J Pharm Pharmacol. 2005; 57: 521-526.

60. Rutland J, De longh RU. Random ciliary orientation: a cause of respiratory tract disease. N Engl J Med. 1990; 323: 1681-1684.

61. Stannard W, Rutman A, Wallis C, O'Callaghan C. Central microtubular agenesis causing primary ciliary dyskinesia. Am J Respir Crit Care Med. 2004; 169: 634-637.

62. Jorissen M, Van der Schueren B, Van den Berghe H, Cassiman JJ. The preservation and regeneration of cilia on human nasal epithelial cells cultured in vitro. Arch Otorhinolaryngol. 1989; 246: 308-314.

63. Jorissen M, Bessems A. Normal ciliary beat frequency after ciliogenesis in nasal epithelial cells cultured sequentially as monolayer and in suspension. Acta Otolaryngol. 1995; 115: 66-70. 
64. Date H, Yamashita M Nagahiro I, et al. Living-donor lobar lung transplantation for primary ciliary dyskinesia. Ann Thorac Surg. 2001; 71: 2008-2009.
Miss Joanne Rimmer

Department of Otolaryngology

Royal National Throat Nose \& Ear

Hospital

London

WC1X 8DA

United Kingdom

Tel: +44-797-4159348

Fax: +44-207-833 9480

E-mail:jrimmer@doctors.org.uk

\section{ADVERTISEMENT}

\section{ERS-FELLOWSHIPS 2013}

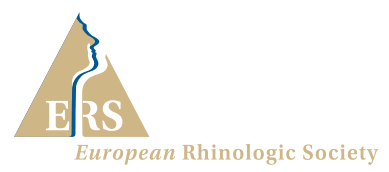

Sponsored by

\section{THE EUROPEAN RHINOLOGIC SOCIETY}

Available to ERS-members from emerging European countries

One place available on each of the following Courses in 2013

10th International Course in Advanced Sinus Surgery

Techniques

Amsterdam, The Netherlands, March 7-8, 2013

Course Director: Prof. W.J. Fokkens

Info: M.B. van Huiden

Fax: +31-20-566 9573

E-mail: m.b.vanhuiden@amc.uva.nl

www.sinuscourse.nl

$45^{\text {th }}$ International Workshop on Functional and Endoscopic Sinus Techniques with Advanced Seminar

Graz, Austria, September 4-7, 2013

Course Director: Prof. Dr. H. Stammberger

E-mail: claire.zwerina@meduni-graz.at

www.endovienna2012.com

$20^{\text {th }}$ International Course in Modern Rhinoplasty

Techniques

Amsterdam, The Netherlands, October 23-25, 2013

Course Director: Dr. D.J. Menger

Fax: +31-20-566 9573

E-mail: m.b.vanhuiden@amc.uva.nl

www.rhinoplastycourse.nl $14^{\text {th }}$ International Course on Reconstructive and Aesthetic Surgery of the Nose and Face "Around the Nose" Nijmegen, The Netherlands, June12-14, 2013

Course Directors: Dr. K. Ingels and N. van Heerbeek Tel +31-24-361 9671

E-mail: Y.Savelkoul-Broekman@pao.umcn.nl www.paoheyendael.nl

Endoscopic approach to Rhinosinusitis in Sinonasal Disease London, United Kingdom, October-2-5, 2013

Course Director: Prof. V.J. Lund

Fax: +44-20-7-837 9279

E-mail: c.overington@ucl.ac.uk

www.ucl.ac.uk/ear/training/short-courses

Sponsorship comprises a sum of $€ 1200$,- to assist in both registration fees and costs of accommodation/travelling. Application forms are available from the Secretary of the ERS (e-mail: rhinologysecretary@amc.uva.nl) or via the website:

\section{http://www.europeanrhinologicsociety.org}

Applications should be submitted before February $1^{\text {st }}, \mathbf{2 0 1 3}$ and directed to the Secretary of the European Rhinologic Society by e-mail: rhinologysecretary@amc.uva.nl

Applicants should be under the age of 40 years, coming from emerging European countries and should be ERS-members without former granting of an ERS-fellowship. 